\title{
$\underline{\mathbf{P}-81}$
}

\section{Standardization and Preliminary Anti-Angiogenic Study of Labisia Pumila}

\author{
Gheniya Ghafar*, Abdalrahim F.A. Aisha, Hooi Kheng Beh, Mohd Shahrul Ridzuan Hamil Zhari \\ Ismail $^{*}$
}

Department of Pharmaceutical Chemistry, School of Pharmaceutical Sciences, Universiti Sains Malaysia, 11800 Minden, Penang, Malaysia; E-mail: gheniyaghafar@yahoo.com

Labisia pumila var. alata (Myrinaceae) locally known as Kacip Fatimah is widely used as traditional medicine in Malaysia. Standardization of herbal preparations is vital to maintain quality, efficacy and safety. This study aims to obtain the phytochemical profile of $L$. pumila and to study the anti-angiogenic effect of the ethanolic, $50 \%$ ethanolic and water extracts of the whole plant. The standardization was done according to the WHO guidelines. Three testing parameters tested including identity (microscopic and TLC profiling), purity (foreign matter, total ash, insoluble acid ash, moisture content and content of extractable matter) and chromatographic and spectroscopic analysis (HPLC, UV-Vis and FT-IR profiling). The rat aortic ring assay was performed to investigate the anti-angiogenic effect of the extracts. Identity of the plant was confirmed by comparing the results with the Malaysian Herbal Monograph Vol.1 (MHM). The purity test results showed studied parameters fall within the normal range of the MHM. HPLC chromatography showed the presence of gallic acid at the retention time of 6.9 min. The maxima absorbance of UV-Vis was at $270 \mathrm{~nm}$ and the main FT-IR bands vibration were obtained at 3335,1604 and $1042 \mathrm{~cm}^{-1}$. The anti-angiogenic test showed $100 \%$ inhibition of microvessels outgrowth in the ethanolic and $50 \%$ ethanolic extracts at 100 $\mu \mathrm{g} / \mathrm{ml}$ whereas the water extract showed $32 \%$ inhibition. The rat aortic rings test showed a possible anti-angiogenic effect which may have interesting applications in the treatment of angiogenesis related disorders.

Keywords: Standardization, Labisia pumila, anti-angiogenic. 